\title{
Demography and Treatment of Complex Regional Pain Syndrome Among Children
}

\author{
Martin Lehmann Boesen*, Anne Estmann Christensen \\ Hans Christensen Children Hospital, Odense University Hospital, Odense, Denmark \\ Email address: \\ lehmannboesen@gmail.com (M. L. Boesen), anne.estmann@dadlnet.dk (A. E. Christensen) \\ ${ }^{*}$ Corresponding author
}

\section{To cite this article:}

Martin Lehmann Boesen, Anne Estmann Christensen. Demography and Treatment of Complex Regional Pain Syndrome Among Children. American Journal of Clinical and Experimental Medicine. Vol. 8, No. 1, 2020, pp. 6-9. doi: 10.11648/j.ajcem.20200801.12

Received: March 2, 2020; Accepted: April 8, 2020; Published: April 29, 2020

\begin{abstract}
Introduction: Complex Regional Pain syndrome (CRPS) is a rare chronic pain syndrome characterized by amplified pain and autonomic dysfunction. A systematic multidisciplinary treatment program for affected children exists at our department. This study aims to estimate the incidence of CRPS and describe the demography, symptoms and psychosocial characteristics of the patients and evaluate the treatment results. Method: A retrospective medical chart review of all patients under the age of 16 years treated for CRPS between 2004 and 2014 at Hans Christian Andersen Children Hospital. Results: A total of 29 children fulfilled the diagnostic criteria for CRPS. The mean incidence was 2,4/100.000 children per year. Most patients were girls $(90 \%)$ with a median age of 11 years. A lower extremity was most often involved (86\%). Time to diagnosis had a mean of 174 days. All patients reported complete disappearance or significant reduction of pain at discharge without any medication. Walking was normalized in $75 \%$ and almost $1 / 3$ could run normally. School attendance and social life was restored. Conclusion: The typical child with CRPS is a girl of normal stature, around 11 years old, who is affected by intolerable pain in a lower extremity and has severe limitation in walking. The condition is rare with a mean incidence of 2,4/100.000 children. Attention is important in order to reduce the diagnostic delay. Our treatment program was successful as the children were in reduced pain, gaining normal physical function and out of social isolation at completion of the program.
\end{abstract}

Keywords: Pain, Children, Musculoskeletal Symptoms, Physiotherapy

\section{Introduction}

Complex regional pain syndrome (CRPS), earlier known as reflex sympathetic dystrophia, is a seldom, but chronic and invalidating pain syndrome that may affect both children and adults.

CRPS is characterized by amplified chronic pain disproportionate with the stimulus to a degree where wearing clothes is impossible. Abnormal vasomotor function with changes in skin color and/or temperature and swelling of the affected area are typical features of CRPS. This makes it different from other chronic pain syndromes. The pain is often localized in a limb and of such intensity that the patient is incapable to fulfill daily activities causing school absence and social isolation $[1-3,16]$.

There are two types of CRPS: One without known nerve injury (type I) and one with nerve injury mostly being the result of an operation (type II). CRPS type I often evolves after immobilization suggesting the need for a triggering factor in susceptible children [4] but the pathogenesis of CRPS remains unknown.

A possible pathogenesis is changes in the small fibers in the peripheral nerves leading to amplified pain sensation. Dysfunction in the peripheral or central nervous system might cause central sensitization $[1,4,5,12,14]$. The psychological aspect of CRPS is uncertain. There are studies pointing to different psychological factors that may influence the susceptibility to CRPS such as stressful life events and tendency to anxiety $[4,15]$. Genetic susceptibility is also described [13].

The diagnosis is clinical and diagnostic criteria have now been established $[7,8]$. 
The treatment of CRPS is multidisciplinary and the prognosis for children with CRPS is favorable in experienced centers where both physiotherapy and psychological support are established parts of the treatment of CRPS $[5,6,16]$. A wide range of treatment modalities with analgesics have been tried over the years [17] but to date pharmacological treatment does not have a place in the international treatment recommendations of childhood CRPS $[4,11]$.

Pain relief at discharge is reported among 90 per cent of the patients $[5,9,10]$. Sherry et al followed children treated for CRPS and found that almost $90 \%$ were still pain free without physical limitations at 5 years follow-up [10].

In this work we focus on CRPS type I among children referred to Hans Christian Andersen Children Hospital (HCA), Odense University Hospital. We aim to estimate the incidence of the condition and describe the demography, symptoms and psycho-social characteristics of the patients. In addition we evaluate the results of treatment in terms of pain reduction, degree of physical limitations and social isolation.

We would like to increase the awareness of CRPS in children, as the syndrome is invalidating due to severe pain, and we want to show that potential curable treatment exists.

We believe that this is the first work to report an incidence, demography and recovery rate for Danish childhood CRPS.

\section{Method}

Hans Christian Andersen Children Hospital is a local hospital for the community with an uptake of 110.000 children, and at the same time the hospital is a tertiary center for pediatric referral from the Region of Southern Denmark.

Since 2004 HCA has employed a systematic multidisciplinary in-patient treatment program for children with CRPS. This method was inspired by treatment principles introduced at The Children's Hospital of Philadelphia, fronted by Dr. David Sherry [10].

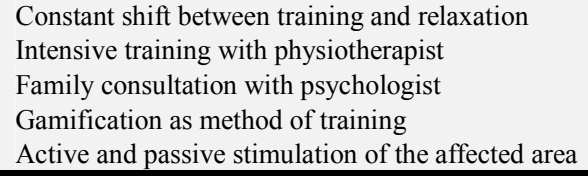

Physiotherapeutic training was carried out daily, performed by a physiotherapist experienced in handling CRPS. After introducing the training program to the child and parents, the intensive training was performed without attendance of the parents. Training sessions guided by nurses, were also performed. Here the child and parent were active together in games such as table tennis, table football, hide and seek etc. Any type of playing, where the affected limp was moved under the distraction of a funny game or competition, was used in these sessions. Each session was typically 30-60 minutes long.

Along with this active training, graded tactile desensitization of the area was performed daily. Brushing with feathers, rolling a soft ball over the area and exposure to cold and warm water, passively stimulated the affected area. This desensitization was performed by a clinical social worker in a quiet and relaxing environment with dimmed light and soft music together with the parent.

The daily training set-up was intense, often causing frustration, tears and fear of failure, why an experienced team set-up was needed to support and guide these children and their families.

During admittance, participation in the hospital school was compulsory. To some patients this was a reintroduction as they had been absent from school for a long period. It enabled the hospital schoolteacher to contact the child's local school, facilitating the return to normal school attendance after discharge. Participation in the school was also important in order to observe the child in another context.

The family consulted a clinical psychologist for guidance to manage pain, frustrations or if relevant, child/parent conflicts. The psychologist also helped identifying and handling stressors in the child's surroundings.

Table 1. Key treatment principles.

Table 2. An example of the daily training program during admittance.

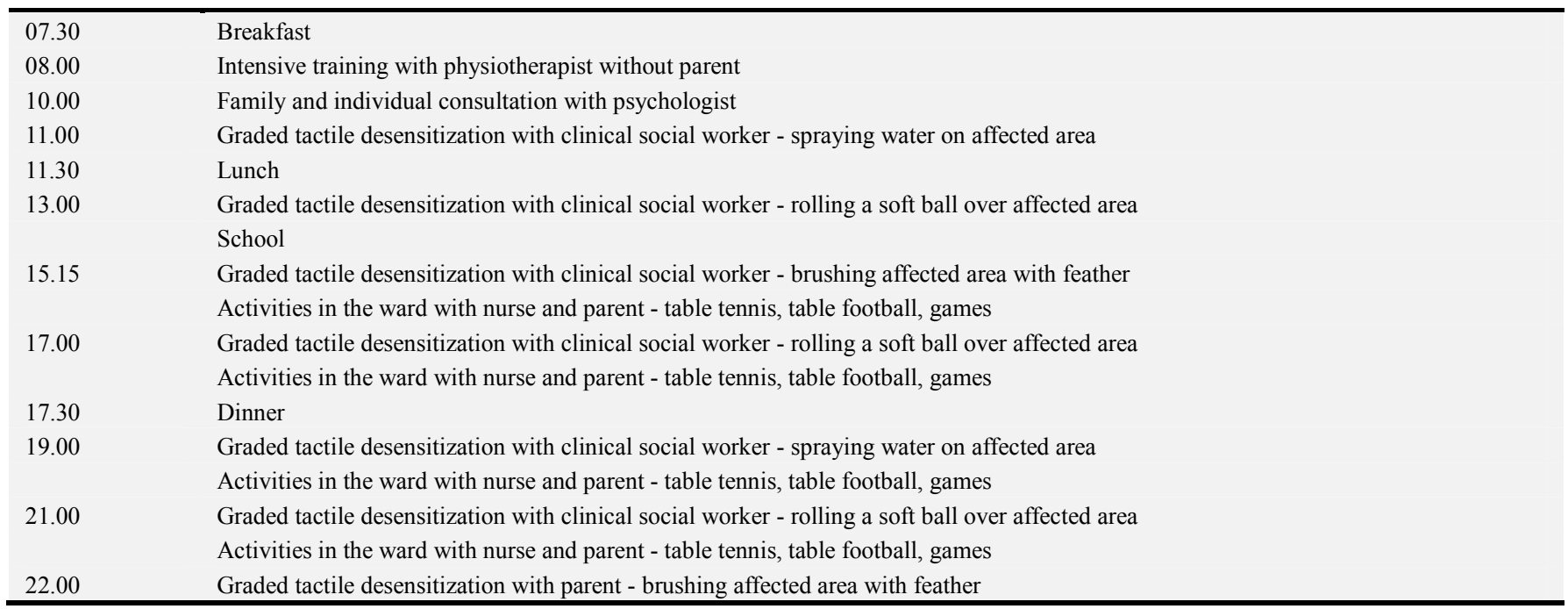


The parent admitted with the child was gradually introduced as the motivator and trainer of all types of training, preparing the family for home training. At discharge they were encouraged and empowered to continue home training 2-4 times a day, and once a week consult the physiotherapist at our out-patient clinic. These consultations usually continued for at least one month.

This is a retrospective work up, of the medical records of children below the age of 16 years diagnosed and treated with CRPS at HCA during eleven years from 2004 to 2014. The work up was carried out in January to March 2015. Medical records were identified using the Danish diagnosing system: Diagnosis Related Group (DRG). Medical records coded as Reflex sympathetic dystrophia (DM890 - ICD-10; M896), Pain in extremities (DM626 to DM796 - ICD-10; M629 to M796) and Chronic pain (DR522 to DR529 - ICD10 ; G90.50 to G90.59) were analyzed and those fulfilling the diagnostic criteria for CRPS were enrolled in the study.

Each journal was analyzed for 52 individual factors related to demography, symptoms, psychosocial characteristics of the child and family, method of referral to our center and diagnostic work up prior to referral. Co-morbidities or known pain syndromes in the families were noted.

Simple descriptive statistics were used.

\section{Results}

From 2004 to December 2014, 29 children were identified fulfilling the diagnostic criteria for CRPS equaling a mean incidence of 2,4 per 100.000 children annually. The incidence rose from 2,1/100.000 in 2004-2009 to 3,4/100.000 annually between 2009 and 2014. The patients were mainly ethnic Danish girls with a median age of 11 years (range 8 years and 8 months to 14 years and 1 month). Their body mass index (BMI) was normal and most had been active athletes prior to their disease, some even at a high level (table 3).

Table 3. Demography of patients.

\begin{tabular}{ll}
\hline Demography & no $(\%)$ \\
\hline Number of patients & $29(100)$ \\
Female & $26(90)$ \\
Ethnic Danish & $28(97)$ \\
CRPS localised to lower limb & $25(86)$ \\
BMI $<20$ & $22(76)$ \\
Active athletes prior to disease & $24(83)$ \\
Elite athlete prior to disease & $3(10)$ \\
& median (range) \\
Age & 11 years $(88 / 12-141 / 12)$ \\
Days of symptoms & 174 days $(1-1280)$ \\
\hline
\end{tabular}

Most patients had localized symptoms from a lower extremity (almost all involving the foot and ankle). Most of them were unable to walk as weight-bearing activity increased the pain to an unbearable level. Over half the patients at admittance used wheel chair or crouches. Absence from school and from social activities was a major concern (table 4). Almost half (46\%) used daily analgesics (paracetamol or non-steroid-anti-inflammatory-drug). Due to incomplete data it was impossible to describe the socioeconomic status of the families.

Eighteen patients $(62 \%)$ were admitted for two weeks or less, $6(21 \%)$ longer and $5(17 \%)$ were treated solely in the out patient clinic. Time from first symptom to first contact at HCA was 174 days (range 1-1280). We found no correlations between length of symptoms and length of admittance.

Children were primarily referred to HCA from general practitioners and orthopedics, and prior to referral an extended examination program had often already been undertaken. Magnetic resonance imaging (MRI) was performed in $52 \%$, X-ray in $58 \%$ and $48 \%$ had consulted a physiotherapist.

At discharge from the hospital ward, all patients indicated that their pain had disappeared completely or was significantly reduced. They had all returned to school and had picked up on their social activities again. No one used analgesics and only one was still using crouches. Almost half the patients could walk normally and some could also run normally. By the end of the training program at the out patient clinic at HCA almost $1 / 3$ could run normally and most $(75 \%)$ could walk completely normal.

One or more regular CRPS setbacks were seen in 6 patients (21\%). Median time for first set back was 275 days (range 29-901). All had symptoms identical to the primary attack and the location of the symptoms was identical. Only one patient required re-admittance - all others managed the condition at home, by physiotherapist-guided self-training. We found no correlation between length of symptoms and number of setbacks.

Interestingly, 7 patients developed a condition of functional pain later on, but not involving the location of their preceding CRPS. Four children later consulted a child psychiatrist.

\section{Discussion}

We found the treatment program for CRPS at HCA successful, as the patients were relieved from or reported considerable improvement in pain and showed almost completely restored physical ability function at discharge.

At our hospital CRPS is a seldom syndrome with an incidence of 2,4 per 100.000. Most patients presented with a lower extremity as the main location of the disease. The most common triad of symptoms was pain in a lower extremity in equivalent to stimuli along with reduced skin temperature and edema of the region. It mainly affected younger girls of normal stature. The majority used to compete in sports and have an active social life, but due to the intense pain they were on the verge of being socially isolated including high rates of school absence.

We found a rather long delay from the appearance of symptoms, to the first contact with HCA - on average almost 6 months.

The incidence of CRPS of 2,4/100.00 annually, might 
underestimate the true incidence. Some children may have been treated in other settings by orthopedics or anesthesiologists. In spite of this, CRPS remains a rather seldom diagnosis. The increase in incidence over the study period might be a result of changes in the referral practice due to increased awareness of the condition or of the treatment possibility at HCA.

The patient characteristics in our cohort were largely identical to that of the literature $[2,6,10,16]$.

The good result using intensive multidisciplinary treatment, align well with other studies, and stresses the need for correct referral to a pediatric department, capable of treating this condition.

This is a retrospective patient journal work up, and data regarding certain questions, such as socio economic status and family history of pain syndromes, were incomplete. The number of set backs we have registered were based on records from our own department, obtained after a different follow-up time for each patient. No information from other health institutions was collected. There is therefore a risk of underreporting reoccurrence of CRPS, but the frequency aligns that of the literature $[7,16]$.

In order to describe the long-term prognosis for CRPS in our patients, a follow up study is needed. This would shed light on the risk of reappearance and on our results indicating that children with CRPS are at risk of developing other pain syndromes later in life.

\section{Conclusion}

We report on incidence, demography and treatment results of childhood CRPS in Denmark. We found an incidence of CRPS on 2,4 per 100.000 annually among children in our uptake area. The typical child with CRPS was a girl of normal stature, around 11 years old who was affected by intolerable pain in a lower extremity, in which there was edema and a decrease in skin temperature. Prior to disease development, the typical child was active socially and physically, but at the time of referral, social isolation was beginning. Our treatment program was successful as the children were in reduced pain, gaining normal physical function and no longer in social isolation at completion of the program. In addition most families had gained knowledge about handling stressors and conflicts.

We hope for an increased awareness of CRPS in order to reduce the diagnostic delay.

\section{References}

[1] Saxton DL, Lindenfeld TN. Complex regional pain syndromes, including reflex sympathetic dystrophy and causalgia. DeLee \& Drez's Orthopaedic Sports Medicine chapter 7, section A, 351-369.
[2] Birklein F. Complex regional pain syndrome. J Neurol 2005; 252: $131-138$.

[3] O'Connell NE et al. Interventions for treating pain and disability in adults with complex regional pain syndrome - an overview of systematic reviews. Cochrane Database of Systematic Reviews 2013; Issue 4.

[4] Weissmann R, Uziel Y. Pediatric complex regional pain syndrome: a review. Pediatric Rheumatology 2016; 14: 29.

[5] Borchers AT, Gershwin ME. Complex regional pain syndrome a critical review. Autoimmunity Reviews 2014; 13: 242-265.

[6] Logan et al. Children and adolescents with complex regional pain syndrome: More psychologically distressed than other children in pain? Pain Res Manag 2013 March/April; 18: 8793.

[7] Merskey H, Bogduk N. Classification of Chronic Pain: Descriptions of Chronic Pain Syndromes and Definitions of Pain Terms 1994; 2nd ed. IASP Press, Seattle.

[8] Wilson P, Stanton-Hicks, Harden RN. CRPS: Current Diagnosis and Therapy. Progress in Pain Research and Management 2005; Vol. 32 IASP Press, Seattle.

[9] Stanton-Hicks. Plasticity of complex regional pain syndrome (CRPS) in children. Pain Med 2010 Aug; 11: 1216-23.

[10] Sherry DD et al. Short- and long-term outcomes of children with complex regional pain syndrome type I treated with exercise therapy. Clinical Journal of Pain 1999; 15: 218-223.

[11] Rodriguez MJ et al. Invasive Management for Pediatric Complex Regional Pain Syndrome: Literature Review of Evidence. Pain Physician 2015 Nov; 18: 621-30.

[12] Lebel A, Becerra L, Wallin D. fMRI reveals distinct CNS processing during symptomatic and recovered complex regional pain syndrome in children. Brain 2008 Jul; 131: 1854-79.

[13] de Rooij AMr, de Mos M, Sturkenboom MC. Familial occurrence of complex regional pain syndrome. Eur J Pain $2009 \mathrm{Feb} ; 13: 171-7$.

[14] Richards S, Chalkiadis G, Lakshman R. Complex regional pain syndrome following immunisation. Arch Dis Child 2012 Oct; 97: 913-5.

[15] Logan DE, Williams SE, Carullo VP. Children and adolescents with complex regional pain syndrome: More psychologically distressed than other children in pain? Pain Res Manag 2013; 18: 87-93.

[16] Mesaroli G et al. Clinical Features of Pediatric Complex Regional Pain Syndrome: A 5-Year Retrospective Chart Review. Clin J Pain. 2019; 35: 933-940.

[17] Cucchiaro G et al. Short- and long-term results of an inpatient programme to manage Complex Regional Pain Syndrome in children and adolescents. Br. J. Pain. 2017 May; 11: 87-96. 